\title{
Iterative Channel Estimation for Higher Order Modulated STBC-OFDM Systems with Reduced Complexity
}

\author{
İlhan Baştürk ${ }^{1,2}$, Berna Özbek ${ }^{2}$ \\ ${ }^{1}$ Electrical and Electronics Engineering Department, \\ Adnan Menderes University, Aydın, Turkey \\ ${ }^{2}$ Electrical and Electronics Engineering Department, \\ Izmir Institute of Technology, İzmir, Turkey \\ [e-mails: ilhan.basturk@adu.edu.tr, bernaozbek@iyte.edu.tr] \\ *Corresponding author: İlhan Baştürk
}

Received October 19, 2015; revised February 8, 2016; revised March 17, 2016; accepted April 20, 2016; published June 30, 2016

\begin{abstract}
In this paper, a frequency domain Expectation-Maximization (EM)-based channel estimation algorithm for Space Time Block Coded-Orthogonal Frequency Division Multiplexing (STBC-OFDM) systems is investigated to support higher data rate applications in wireless communications. The computational complexity of the frequency domain EM-based channel estimation is increased when higher order constellations are used because of the ascending size of the search set space. Thus, a search set reduction algorithm is proposed to decrease the complexity without sacrificing the system performance. The performance results of the proposed algorithm is obtained in terms of Bit Error Rate (BER) and Mean Square Error (MSE) for 16QAM and 64QAM modulation schemes.
\end{abstract}

Keywords: Channel estimation, space-time block codes, OFDM, expectation-maximization algorithm

A preliminary version of this paper appeared in IEEE WCNC 2009, April 5-8, Budapest, Hungary. This version proposes a search set reduction algorithm for the higher order modulation schemes in order to decrease the computational complexity. 


\section{Introduction}

F or wireless communication systems, orthogonal frequency division multiplexing (OFDM) which has been already involved in many wireless communication standards is one of the promising modulation technique because of its ability to combat inter-symbol interference (ISI) over multipath fading channels [1]. Transmitter diversity is an effective technique for combating fading in mobile wireless communications. A simple and powerful diversity technique using two transmit antennas has been proposed by Alamouti [2] and its various derivatives have been developed in [3][4]. Combining OFDM systems with these multiple antenna techniques, the system performance and robustness against fading can be improved.

An accurate channel estimation for OFDM systems is required in order to demodulate the data coherently. Pilot-based channel estimation algorithms are widely used in the literature because of their reliability and lower complexity. There are many studies for the single-input single-output (SISO)-OFDM case using these algorithms [5][6]. These algorithms cannot be simply extended to the multiple antenna scenarios, since the received signal is the sum of the signals transmitted from all the transmit antennas and each subchannel at the receiver is associated with multiple channel parameters. In [7]-[9], pilot-based channel estimation algorithms have also been studied for Space Time Block Coded (STBC)-OFDM systems. Because of the limited performance of the pilot based channel estimation algorithms, the Expectation-Maximization (EM) algorithm defined in detail in [10]-[12], is utilized to improve the channel estimation performance through iterations in the literature. For SISOOFDM case, three different EM-based algorithms for channel estimation have been compared in [13]. Two of them estimate the channel frequency response and the channel impulse response iteratively which are called as frequency and time domain EM-based algorithms respectively, and the third one estimates directly the transmitted symbols using the channel impulse response statistics. The third algorithm has been used in [14] for STBCOFDM systems. However, this algorithm has higher complexity and lower convergence time and also assumes that statistics such as mean and covariance are known which may not be valid for practical applications. A time domain EM-based algorithm has been applied to Space-Time Trellis Coded (STTC)-OFDM systems in [15]. This algorithm has been modified for STBC-OFDM and Space Frequency Block Coded (SFBC)-OFDM in [16] and STBC-OFDM uplink multiuser case in [17]. All these time domain algorithms for space-time codes are followed by the decomposition of the superimposed signals [18]. While decomposing the received signal, the noise is also being decomposed with a coefficient which must be chosen correctly to obtain good performances. Moreover, these algorithms require a matrix inversion while updating the channel coefficients which increase the computational complexity. In [19], we have proposed a frequency domain EM-based channel estimation algorithm for STBC-OFDM systems to eliminate the drawbacks of the time domain EM-based channel estimation algorithms.

All these existing EM-based channel estimation algorithms including [19] in the literature are only performed for low order constellations which is suitable for low data rate applications. However, nowadays wireless communication systems require higher data rates achieved by using higher order modulation schemes. Not only the data rate of the system but also the complexity of the channel estimation process of the EM-based algorithm increases significantly with the order of constellations since the EM algorithm is an iterative solution for Maximum Likelihood (ML) and causes an increase in the number of points to be search. 
In the literature, different methods such as sphere decoding (SD) [20]-[22] and two-stage detection [23]-[26] have been presented to be able to reach close ML performance by lowering the computational complexity. However, these works have focused on the ML detection problem and they have ignored the channel estimation. These works also have not considered EM algorithm to solve the ML detection problem. Thus, to the best of our knowledge there is a gap in the literature to solve the complexity problem of the higher order modulated EM-based channel estimation algorithms without performance loss.

In this paper, we propose a search set reduction algorithm to reduce the complexity of the frequency domain EM-based channel estimation for STBC-OFDM systems without sacrificing the performance for higher order modulation schemes. The contribution and features of the proposed scheme are summarized as follows:

- The proposed frequency domain channel estimation scheme for STBC-OFDM systems in [19] is extended to the higher order constellations in order to satisfy the high data rate requirements of the future wireless communication systems.

- Increasing the constellation points also increases the system complexity, thus, a search set reduction scheme in which reduced number of constellation points are used instead of searching the entire constellation points is proposed.

- The proposed channel estimation scheme is more applicable for the practical systems since the computational complexity is reduced while providing almost the same performance.

The rest of this paper is organized as follows. Section 2 introduces the STBC-OFDM system model. Section 3 describes the proposed low complexity frequency domain EMbased channel estimation scheme for STBC-OFDM with higher order constellations. Section 4 provides simulation results to evaluate the proposed channel estimation scheme. The strength of the proposed low complexity scheme for higher order modulations is revealed by comparing it to the frequency domain full search case and time domain case in terms of the BER and MSE results. The conclusion is given in Section 5.

\section{System Model for STBC-OFDM}

In this paper, the Alamouti STBC-OFDM system model with two transmit antennas and one receive antenna is used as shown in Fig. 1. At time $n$, a data block $S(n, k), k=$ $0,1,2, \ldots, K-1$, where $K$ is the number of subchannels, is encoded into two different symbol blocks, $X_{i}(n, k)$ with $i=1,2$. These blocks can be written for two consecutive times in Eq. (1) as follows:

$$
\begin{aligned}
& X_{1}(n, k)=S(n, k) \\
& X_{2}(n, k)=S(n+1, k) \\
& X_{1}(n+1, k)=-S(n+1, k)^{*} \\
& X_{2}(n+1, k)=S(n, k)^{*}
\end{aligned}
$$

where $n=0,2,4, \ldots, N-1$ and $(\cdot)^{*}$ denotes complex conjugate.

The STBC-OFDM symbols are transmitted through the frequency selective fading channel after performing Inverse Fast Fourier Transform (IFFT) and adding cyclic prefix (CP) to eliminate ISI. Then, applying a Fast Fourier Transform (FFT) and removing CP, the received signal is obtained as a superposition of transmitted signals as: 


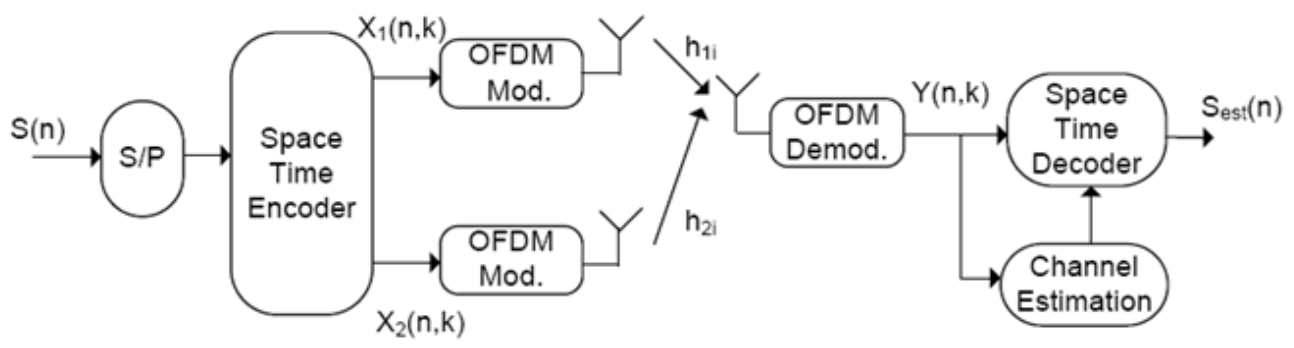

Fig. 1. STBC-OFDM System Model

$$
Y(n+j, k)=\sum_{i=1}^{2} X_{i}(n+j, k) H_{i}(n+j, k)+N(n+j, k)
$$

where $N(n+j, k)$ is the additive white Gaussian noise with zero mean and variance $\sigma^{2}$, $H_{i}(n+j, k)$ denotes the channel frequency response of the multipath channel belongs to $k$ th subchannel and $i$ th antenna and $j=0,1$. The channel coefficients between two consecutive times are assumed to be quasi-static and satisfies $H_{i}(n, k)=H_{i}(n+1, k)=H_{i}(k)$. Thus, the decoded symbols at the output of the space time decoder is given as;

$$
\begin{aligned}
\tilde{S}(n, k) & =H_{1}^{*}(k) Y(n, k)+H_{2}(k) Y^{*}(n+1, k) \\
\tilde{S}(n+1, k) & =H_{2}^{*}(k) Y(n, k)-H_{1}(k) Y^{*}(n+1, k)
\end{aligned}
$$

Accurate estimation of channel parameters is the key for decoding of the space-time codes, therefore channel estimation algorithms will be examined in detail in the following sections.

\section{Channel Estimation Algorithms for STBC-OFDM}

\subsection{EM Algorithm Basics}

The EM algorithm is an iterative method for solving the ML estimation problems in the presence of unobserved data. The aim of this algorithm is to augment the observed data with the hidden data so that it will be easy to manipulate the likelihood function conditioned on the data and the hidden data. The algorithm consists of two major steps: an expectation step (E-step) followed by a maximization step (M-step) [11][12]. We can divide our complete data into two components such as $\Gamma=(\Pi, \gamma)$, where $\Pi$ are the observed data or incomplete data and $Y$ are the hidden data or the missing data. We estimate an unknown paramater denoted as $\theta$ by using the missing data $Y$.

According to the EM procedure E-step finds $\Theta\left(\theta \mid \theta^{(a)}\right)$, the expected value of the loglikelihood of the $\theta$. The expectation is taken with respect to $Y$ conditioned on $\Pi$ and $\theta^{(a)}$ which the latest estimate of the $\theta$ : 


$$
\Theta\left(\theta \mid \theta^{(a)}\right)=E\left\{\log f(\Gamma \mid \theta) \mid \Pi, \theta^{(a)}\right\}
$$

where $a=0,1,2, \ldots$ is the iteration number. We can find the $\theta^{(a+1)}$, which maximizes $\theta^{(a)}$ over all possible values of $\theta$,

$$
\theta^{(a+1)}=\arg \max _{\theta} \Theta\left(\theta \mid \theta^{(a)}\right)
$$

These two steps are iterated until $\theta^{(a)}$ converges to the ML estimate of $\theta$.

This algorithm has been used in many areas such as genetics, econometric, clinical, sociological studies, signal processing and communication applications. The definiton of complete and incomplete data sets can be defined according to the usage areas and applications. The reader is referred to [11][12] for details on the EM algorithm. In this study, EM algorithm is applied to wireless communications systems in order to estimate the channel coefficients. Thus, we use the received and transmitted signals together $(\boldsymbol{Y}, \boldsymbol{X})$ as the complete data set and the received signal $\boldsymbol{Y}$ is the incomplete data set. Since our application is channel estimation, the unknown parameter is the channel frequency response $\boldsymbol{H}$. The EM algorithm is an iterative algorithm so an appropriate initial channel estimation value $\boldsymbol{H}^{(0)}$ is required. Thus, pilot symbols are used to estimate the initial channel coefficients of the EM algorithm efficiently due to its low computational complexity as explained in the next subsection.

\subsection{Initial Channel Estimation using Pilot Symbols}

In this part, pilot-based channel estimation that is used for the initial channel estimation will be given in detail. The pilot symbols are distributed at certain locations in the OFDM timefrequency grid to estimate the channel coefficients. The channel estimation for STBCOFDM is performed by transmitting pilot symbols from different antennas at the same subcarrier simultaneously. Therefore, the received signal at the pilot positions are the superposition of the signals coming from different transmit antennas which makes estimation issue difficult. Thus, transmitted pilot symbols are encoded using Space Time Block Codes in order to simplify the channel estimation [8].

The pilot symbols are arranged in the OFDM time-frequency grid for two antennas as given in Fig. 2. At time $n$ the pilot symbols $p 1$ and $p 2$, at time $n+1$ the pilot symbols $-p 2^{*}$ and $p 1^{*}$ are sent from the first and second antennas, respectively. The received signal at the pilot symbol positions can be expressed in matrix form as:

where

$$
\boldsymbol{Y}_{p}=\boldsymbol{X}_{p} \boldsymbol{H}_{p}+\boldsymbol{N}_{p}
$$

$$
\begin{aligned}
& \left.\boldsymbol{Y}_{p}=Y\left(n, k_{p}\right) \quad Y\left(n+1, k_{p}\right)\right]^{T} \\
& \boldsymbol{X}_{p}=\left[\begin{array}{cc}
X_{1}\left(n, k_{p}\right) & X_{2}\left(n, k_{p}\right) \\
-X_{2}\left(n, k_{p}\right)^{*} & X_{1}\left(n, k_{p}\right)^{*}
\end{array}\right] \\
& \boldsymbol{H}_{p}=\left[\begin{array}{ll}
H_{1}\left(k_{p}\right) & H_{2}\left(k_{p}\right)
\end{array}\right]^{T} \\
& \boldsymbol{N}_{p}=\left[N\left(n, k_{p}\right) N\left(n+1, k_{p}\right)\right]^{T} \text {. }
\end{aligned}
$$

In Eq. (6), $k_{p}$ is the subcarrier index represents the pilot positions, $Y\left(n, k_{p}\right)$ and $Y(n+$ 
$\left.1, k_{p}\right)$ are the received signals at time $n$ and $n+1$, respectively. The STBC-OFDM system assumes that $H_{i}\left(n, k_{p}\right)=H_{i}\left(n+1, k_{p}\right)=H_{i}\left(k_{p}\right), n=0,2,4, \ldots,(N-1)$, and $i=1,2$ which means the channel is quasi-static for two consecutive times. Then, the estimated channel coefficients at pilot positions can be obtained as,

$$
\begin{aligned}
\widehat{H}_{1}\left(k_{p}\right) & =\frac{1}{2}\left(Y\left(n, k_{p}\right) X_{1}^{*}\left(n, k_{p}\right)-Y\left(n+1, k_{p}\right) X_{2}\left(n, k_{p}\right)\right) \\
\widehat{H}_{2}\left(k_{p}\right) & =\frac{1}{2}\left(Y\left(n, k_{p}\right) X_{2}^{*}\left(n, k_{p}\right)+Y\left(n+1, k_{p}\right) X_{1}\left(n, k_{p}\right)\right)
\end{aligned}
$$

where the pilot signal power is normalized to 1 .

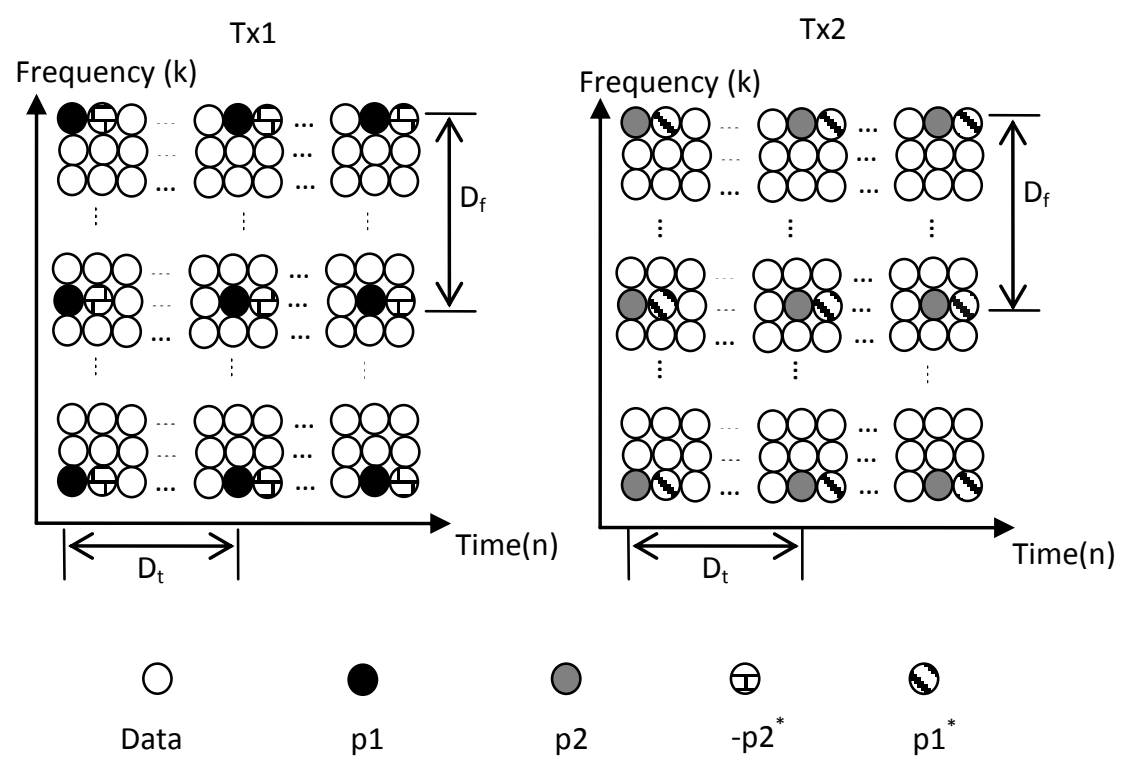

Fig. 2. Pilot Symbol Pattern for one STBC-OFDM frame

The estimated channel vectors can be reconstructed for each OFDM frame and for each antenna $i=1,2$,

$$
\widehat{\boldsymbol{H}}_{i}=\left[\widehat{H}_{i}(1), \widehat{H}_{i}\left(D_{f}+1\right), \ldots, \widehat{H}_{i}\left(\left(N_{f}-1\right) D_{f}+1\right)\right]^{T}
$$

where $D_{f}$ is the distance between the subcarriers and $N_{f}$ is the number of pilot symbols in the frequency axes. We use Discrete Fourier Transform (DFT)-based interpolation technique in the frequency axes in order to estimate all channel coefficients belonging to all subcarriers. First, we transform the frequency channel estimates $\widehat{\boldsymbol{H}}_{i}$ into time domain as follows:

$$
\widehat{\boldsymbol{h}}_{i}=\boldsymbol{F}^{-1} \widehat{\boldsymbol{H}}_{i}
$$

where $\boldsymbol{F}$ is the $N_{f} \times N_{f}$ DFT matrix.

In the pilot arrangement, $N_{f}$ can be selected as greater or equal to the channel length, $N_{f} \geq L_{f}$, where $L_{f}$ is the length of channel. Thus we apply a filtering matrix to $\widehat{\boldsymbol{h}}_{i}$ assuming 
that the channel response is limited to $L_{f}$ and obtain the filtered channel response:

$$
\widehat{\boldsymbol{h}}_{i}^{(0)}=\boldsymbol{W} \widehat{\boldsymbol{h}}_{i}
$$

For the above equations the filtering matrix $\boldsymbol{W}$, is the $L_{f} \times N_{f}$ identity matrix. $\boldsymbol{W}$ can be in the form of square $\left(N_{f}=L_{f}\right)$ or rectangular $\left(N_{f}>L_{f}\right)$ identity matrix. Then we apply DFT in order to obtain the initial estimates for the $n$th OFDM symbol as follows:

$$
\widehat{\boldsymbol{H}}_{i}^{(0)}=\boldsymbol{V} \widehat{\boldsymbol{h}}_{i}^{(0)}
$$

where $\boldsymbol{V}$ is the $K \times L_{f}$ matrix obtained from the first $L_{f}$ columns of the $K \times K$ DFT matrix.

In order to estimate the other channel coefficients in the OFDM frame, we simply apply linear interpolation in the time domain using the estimated channel coefficients for pilot OFDM symbols.

\subsection{The Proposed EM-based Channel Estimation Scheme}

In [19], we have proposed a frequency domain EM-based channel estimation algorithm for low order modulation schemes and shown the superiority of this algorithm over the time domain EM-based channel estimation algorithms. However, the demand on higher data rates is directed us to employ higher order modulation schemes. Although, using the higher order constellations increase the data rate, it also increases the complexity of the channel estimation process because of the increasing number of points to be searched in the EM algorithm. Thus, in this paper, we propose to reduce the number of points to be searched in EM algorithm without sacrificing the performance of the channel estimation.

Using the Gaussian noise assumption, we can calculate the probability density function (pdf) of $Y$ (incomplete data) given $\boldsymbol{X}$ (missing data) and $\boldsymbol{H}$ (unknown parameter vector we try to estimate) for two consecutive times $n$ and $n+1$ as;

$$
\begin{gathered}
f(Y(n, k) \mid \boldsymbol{X}, \boldsymbol{H})=\frac{1}{2 \pi \sigma^{2}} \exp \left\{-\frac{1}{2 \sigma^{2}}|\Phi(1)|^{2}\right\} \\
f(Y(n+1, k) \mid \boldsymbol{X}, \boldsymbol{H})=\frac{1}{2 \pi \sigma^{2}} \exp \left\{-\frac{1}{2 \sigma^{2}}|\Phi(2)|^{2}\right\}
\end{gathered}
$$

where

$$
\Phi=\boldsymbol{Y}-\boldsymbol{X} \boldsymbol{H}
$$

with $\Phi(i)$ is the $i$ th element of the $\Phi, \boldsymbol{Y}=\left[\begin{array}{lll}Y(n, k) & Y(n+1, k)\end{array}\right]^{T}, \boldsymbol{H}=\left[\begin{array}{ll}H_{1}(k) & H_{2}(k)\end{array}\right]^{T}$ and $\boldsymbol{X}=\left[\begin{array}{cc}X_{1}(n, k) & X_{2}(n, k) \\ -X_{2}^{*}(n, k) & X_{1}^{*}(n, k)\end{array}\right]$.

In Eq. (12) and Eq. (13), we need to know $\boldsymbol{X}$ matrix, which contains the symbol pairs $X_{1}(n, k)$ and $X_{2}(n, k)$ transmitted from the first and second antennas, respectively. Although, the exact transmitted signals are not known, the possible symbol pairs can be constructed according to to the modulation type and the number of transmitter antennas. The number of all probable symbol pairs can get $C$ different values that is calculated as $M^{N_{t}}$ in which $M$ and $N_{t}$ represent the size of the constellation points and the number of transmit 
antennas, respectively. For example, if the modulation type is 16QAM and the number of transmitter antennas is $N_{t}=2$, then the constellation size is $M=16$, and the number of the symbol pairs becomes $C=16^{2}=256$. Increasing the modulation order or number of transmitter antennas will increase not only the $C$ value but also the complexity significantly.

By using the assumption that all probable symbol pairs are equally likely and averaging the conditional pdf values given in Eq. (12) and Eq. (13) over the $\boldsymbol{X}$, the pdf of $Y(n, k)$ and $Y(n+1, k)$ for given $\boldsymbol{H}$ is calculated as follows;

Time $n$ :

$$
f(Y(n, k) \mid \boldsymbol{H})=\frac{1}{2 \pi \sigma^{2} C} \sum_{l=1}^{C} \exp \left\{-\frac{1}{2 \sigma^{2}}\left|\Phi_{l}(1)\right|^{2}\right\}
$$

Time $n+1$ :

$$
f(Y(n+1, k) \mid \boldsymbol{H})=\frac{1}{2 \pi \sigma^{2} C} \sum_{l=1}^{C} \exp \left\{-\frac{1}{2 \sigma^{2}}\left|\Phi_{l}(2)\right|^{2}\right\}
$$

where

$$
\Phi_{l}=\boldsymbol{Y}-\boldsymbol{X}_{l} \boldsymbol{H}
$$

and $\boldsymbol{X}_{l}$ is a matrix which take $C$ different values through a set contains all probable symbols.

As mentioned earlier the EM algorithm has two steps: E-Step and M-Step, that are performed in an iterative manner. Our aim is to estimate $\boldsymbol{H}$ from observations of $\boldsymbol{Y}$, given an initial estimate $\boldsymbol{H}^{(0)}$ obtained in the initial channel estimation phase by using pilot symbols. At time $n$, we can estimate $\boldsymbol{H}$ from $Y(n, k)$ at each iteration $(a=0,1,2, \ldots A)$ as the following two steps:

E-Step:

$$
\begin{aligned}
\Theta\left(\boldsymbol{H} \mid \boldsymbol{H}^{(a)}\right) & =E_{\boldsymbol{X}}\left\{\log f(Y(n, k), \boldsymbol{X} \mid \boldsymbol{H}) \mid Y(n, k), \boldsymbol{H}^{(a)}\right\} \\
& =\sum_{l=1}^{C} \log \left\{\frac{1}{C} f\left(Y(n, k) \mid \boldsymbol{H}, \boldsymbol{X}_{l}\right)\right\} \times f\left(\boldsymbol{X}_{l} \mid Y(n, k), \boldsymbol{H}^{(a)}\right) \\
& =\sum_{l=1}^{C} \log \left\{\frac{1}{C} f\left(Y(n, k) \mid \boldsymbol{H}, \boldsymbol{X}_{l}\right)\right\} \times \frac{f\left(Y(n, k) \mid \boldsymbol{H}^{(a)}, \boldsymbol{X}_{l}\right) f\left(\boldsymbol{X}_{l} \mid \boldsymbol{H}^{(a)}\right)}{f\left(Y(n, k) \mid \boldsymbol{H}^{(a)}\right)} \\
& =\sum_{l=1}^{C} \log \left\{\frac{1}{C} f\left(Y(n, k) \mid \boldsymbol{H}, \boldsymbol{X}_{l}\right)\right\} \times \frac{f\left(Y(n, k) \mid \boldsymbol{H}^{(a)}, \boldsymbol{X}_{l}\right)}{C f\left(Y(n, k) \mid \boldsymbol{H}^{(a)}\right)}
\end{aligned}
$$

where $\log f\left(Y(n, k), \boldsymbol{X}_{l} \mid \boldsymbol{H}\right)=\log f\left(Y(n, k) \mid \boldsymbol{X}_{l}, \boldsymbol{H}\right)+\log f\left(\boldsymbol{X}_{l} \mid \boldsymbol{H}\right)$. Since channel impulse response is assumed to be independent of the transmitted symbols, $\log f\left(\boldsymbol{X}_{l} \mid \boldsymbol{H}\right)=$ $\log f\left(\boldsymbol{X}_{l}\right)=\frac{1}{C}$. 
M-Step:

$$
\widehat{\boldsymbol{H}}^{(a+1)}=\arg \max _{\boldsymbol{H}} \Theta\left(\boldsymbol{H} \mid \boldsymbol{H}^{(a)}\right)
$$

The same procedure is also applied at time $n+1$ for estimating $\boldsymbol{H}$ from $Y(n+1, k)$. The expression given in Eq. (19) is differentiated with respect to $\boldsymbol{H}$ for two consecutive time and set to zero to obtain updated channel coefficients. Then, combining these updated channel coefficients obtained at time $n$ and $n+1$, we get:

$$
\widehat{\boldsymbol{H}}^{(a+1)}=\left[\sum_{l=1}^{C} \boldsymbol{X}_{l}^{*} \boldsymbol{X}_{l} \alpha_{l}\right]^{-1}\left[\sum_{l=1}^{C} \boldsymbol{X}_{l}^{*} \boldsymbol{Y} \alpha_{l}\right]
$$

where $\alpha_{l}$ is a scalar value and $\widehat{\boldsymbol{H}}^{(a+1)}=\left[\begin{array}{ll}\widehat{H}_{1}^{(a+1)} & \widehat{H}_{2}^{(a+1)}\end{array}\right]^{T}$.

In Eq. (20), the value of $\alpha_{l}$ is an important parameter to converge the actual channel coefficient values. In considered STBC-OFDM model, this parameter can have two different values for each subchannel, since the symbols and their complex conjugate values are transmitted in two consecutive times. These $\alpha_{l}$ values which belong to two consecutive times are the elements of the $\boldsymbol{G}_{l}$ vector formed by using Eqs. (12)-(16) as given below:

$$
\boldsymbol{G}_{l}=\left[\begin{array}{ll}
\frac{f\left(Y(n, k) \mid \boldsymbol{H}^{(a)}, \boldsymbol{X}_{l}\right)}{f\left(Y(n, k) \mid \boldsymbol{H}^{(a)}\right)} & \frac{f\left(Y(n+1, k) \mid \boldsymbol{H}^{(a)}, \boldsymbol{X}_{l}\right)}{f\left(Y(n+1, k) \mid \boldsymbol{H}^{(a)}\right)}
\end{array}\right]^{T}
$$

The channel coefficients between two consecutive times are assumed to be quasi-static as mentioned before. Thus, only one $\alpha_{l}$ value must be selected through $\boldsymbol{G}_{l}$ vector. In order to improve the confidence of the estimation, the worst case scenario is considered and for each $l$, we choose the lowest case as in [19]:

$$
\alpha_{l}=\min \left\{G_{l}(1), G_{l}(2)\right\}
$$

In Eq. (20), it is seen that the updated channel coefficients are related to $\boldsymbol{X}_{\boldsymbol{l}}$ matrix which contains the transmitted symbols from the first and second antennas. As mentioned above, these symbol pairs so the $\boldsymbol{X}_{l}$ matrix can take $C$ different values. The value of $C$, which is dependent on the modulation type and the number of transmitter antennas of the system, is critical since the higher values of these parameters increase the complexity of the system. In order to support higher data rate applications in wireless communications systems, the higher order constellation schemes are employed with the support of multiple antenna techniques. However, using higher order modulation schemes not only increase the data rate of the system but also increase the complexity of the frequency domain EM-based channel estimation for STBC-OFDM systems significantly.

A lower complexity scheme is required for wireless systems with high data rate systems. Thanks to the proposed low complexity frequency domain channel estimation scheme properties, it is possible to reduce the size of search set by using the initial soft estimates of the transmitted signals, which are obtained by using the received symbols and initial channel estimation coefficients in Eq. (3), as the symbol pairs. The effective lower complexity constellation reduction procedure can be summarized in two steps as given below: 
$\underline{\text { Step } 1}$

- Divide the whole constellation map into lower order constellation regions.

- Determine the reference points for each divided region.

- Decide the approximate regions for the transmitted symbol pairs by comparing the real and imaginary parts of them with these reference points.

$\underline{\text { Step } 2}$

- Discard the unselected regions while forming the $\boldsymbol{X}_{l}$ matrix. Only the constellation points in the selected regions are considered for the evaluation since, these points are more significative than the points in the other regions. This will reduce the value of $C$ for the proposed channel estimation algorithm.

We consider 16QAM and 64QAM as high order modulation schemes which are examined in detail as follows.

\section{QAM case study}

In 16QAM case, constellation map is divided into four regions as given in Fig. 3. The region boundaries are simple for 16QAM that the big green circle $((0,0)$ point $)$ is taken as a reference point. In the first step, the regions of the transmitted symbol pairs $\left(X_{1}(n, k)\right.$, $\left.X_{2}(n, k)\right)$ are determined by using this reference point. The real and imaginary parts of the given symbol pair is compared to reference point to determine the approximate regions. In this step, since we have four regions, we can think each region as a constellation point so the constellation points are 4 instead of 16 for each symbol. Therefore, the search set size will be $C_{16}^{\text {step } 1}=4^{2}=16$. As shown in the Fig. 3, after checking 16 probable region pairs, (Region4, Region3) is selected. In the second step, the regions of the symbol pairs are known so the constellation points belonging to not selected regions such as Region1 and Region2 are ignored while forming the $\boldsymbol{X}_{l}$ matrix. Thus, the constellation points for each symbol are reduced from $M=16$ to $M=4$ for the second step and the search set size became $C_{16}^{\text {step } 2}=$ $4^{2}=16$. Total calculations for the proposed algorithm for 16QAM can be given as $C_{16}=C_{16}^{\text {step } 1}+C_{16}^{\text {step } 2}$. As a result, the overall effect of using regions is reducing the number of calculations from $C=16^{2}=256$ (full search case) to $C_{16}=16+16=32$.

\section{QAM case study}

The constellation map is divided into sixteen regions for 64QAM case as seen in Fig. 4. In the first step, the region of symbol pairs are determined approximately by using the five boundary points (green big circles) whose locations on the constellation map are given as $(0,0),(0.6172,0.6172),(0.6172,-0.6172),(-0.6172,0.6172)$ and $(-0.6172,-0.6172)$. The real and imaginary parts of the symbols are compared with the given boundary points in order to find the approximate regions of the symbol pairs. In this step, since we have 16 regions, we can assume that the constellation points are 16 instead of 64 for each symbol so the search set size will be $C_{64}^{\text {step } 1}=16^{2}=256$. As shown in the Fig. 4, after checking 256 probable regions, (Region13, Region12) which is surrounded with red boxes is selected for $\left(X_{1}(n, k), X_{2}(n, k)\right)$ symbol pair. In the second step, the constellation points belonging to not selected regions are ignored while forming the $\boldsymbol{X}_{l}$ matrix as in the 16QAM case. Thus, the constellation points for each symbol are reduced from $M=64$ to $M=4$ for the second step and the search set size became $C_{64}^{\text {step } 2}=4^{2}=16$. Total calculations for the proposed 
algorithm for 64QAM can be given as $C_{64}=C_{64}^{\text {step } 1}+C_{64}^{\text {step } 2}$. Finally, the calculations for 64QAM case is reduced from $C=64^{2}=4096$ (full search case) to $C_{64}=256+16=$ 272. Due to this, the complexity and process time of the proposed channel estimation is reduced significantly.

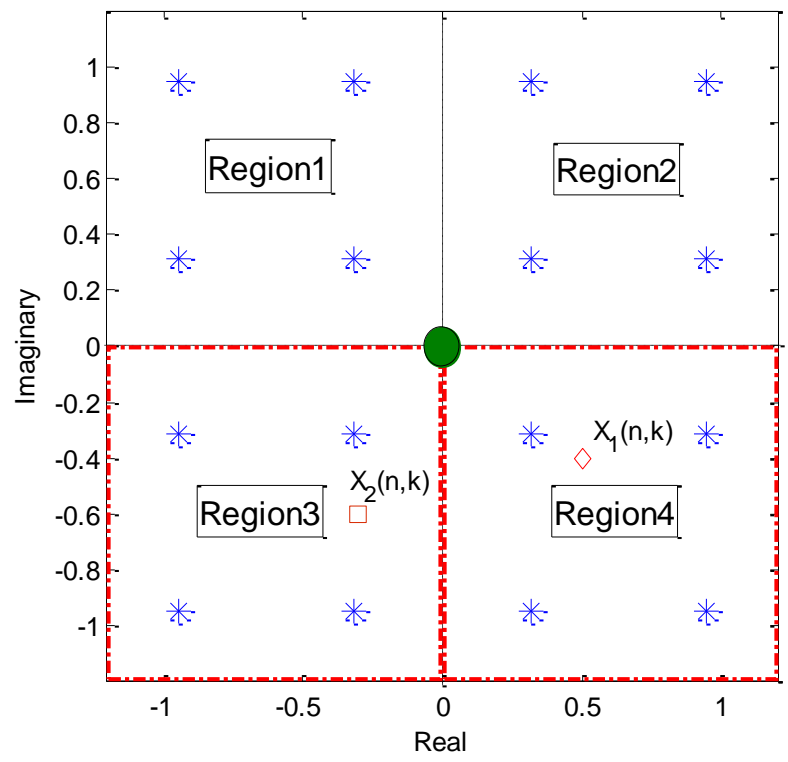

Fig. 3. Regions for 16QAM.

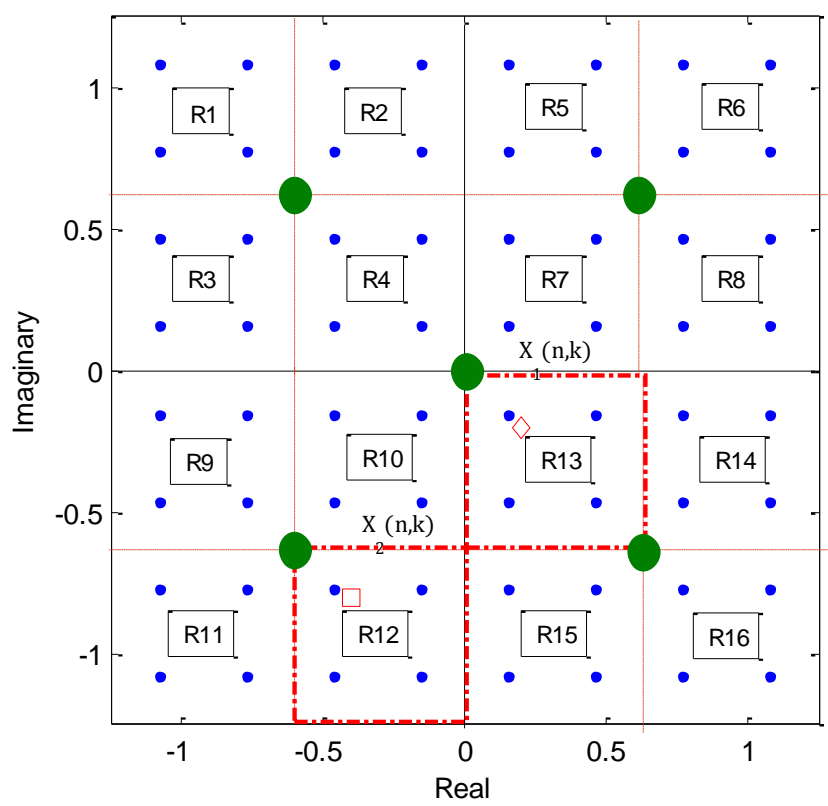

Fig. 4. Regions for 64QAM. 


\section{Performance Results}

In this section, we evaluate the performance of the proposed low complexity frequency domain EM-based channel estimation algorithm for Alamouti STBC-OFDM with higher order constellations. The proposed low complexity iterative channel estimation scheme is compared to the pilot based initial channel estimation, the time domain EM-based STBCOFDM channel estimation algorithm described in [16][17], the frequency domain EM-based STBC-OFDM channel estimation algorithm that searches all constellation points also called full search case and perfect channel state information case for 16QAM and 64QAM in terms of BER and MSE. The BER and MSE are obtained through Monte-Carlo simulations.

In the simulations, the symbol duration is selected as $100 \mu$ s including $20 \mu \mathrm{s}$ guard interval to avoid ISI due to channel delay spread. The entire channel bandwidth is chosen $800 \mathrm{kHz}$, and is divided into 64 subchannels. The time varying frequency fading channel has 8 taps and the Doppler shift is chosen to be $100 \mathrm{~Hz}$. Each tap is Rayleigh distributed and the conventional exponential decay multipath channel model is used for power-delay profile. The channel is assumed to be constant in two consecutive OFDM symbols. We use 64 OFDM symbols in one OFDM frame and 8 pilot symbols for each OFDM symbol $\left(N_{f}=8\right)$. The simulation results are averaged over 2000 simulation runs.

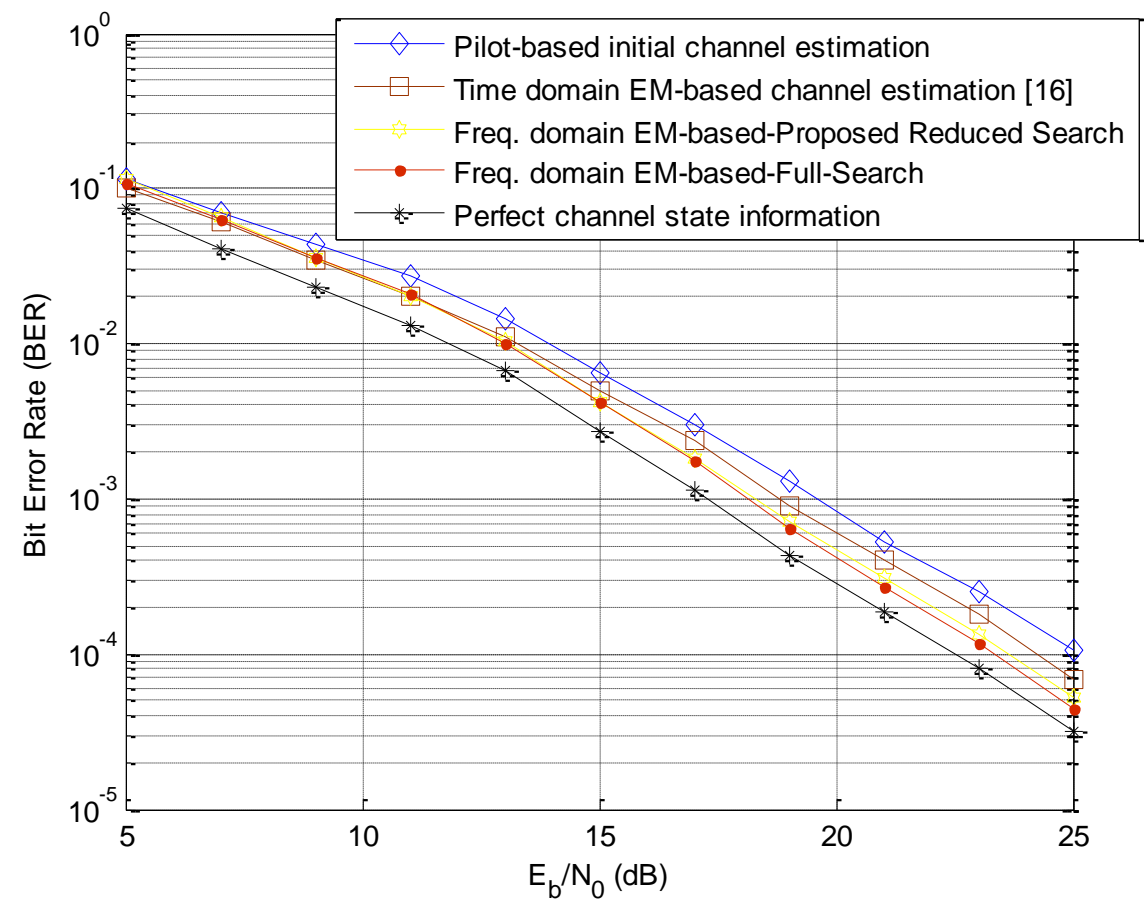

Fig. 5. The BER performance of STBC-OFDM for 16QAM

First of all, we obtained the BER and MSE results in Fig. 5 and Fig. 6, respectively for the 16QAM case. In Fig. 5, it is seen that the EM-based channel estimation algorithms outperform the pilot-based initial channel estimation algorithm. The frequency domain EMbased channel estimation algorithms have also better BER performance compared to the time 
domain EM-based channel estimation algorithm and both algorithms give close performance to the perfect channel estimation case. Although, the proposed low complexity algorithm does not search all possible symbol pairs while estimating the channel coefficients by using EM algorithm, it still has almost the same BER performance with the full search case that search all possible symbol pairs. The total number of symbol pairs so the total calculation value is $C=256$ for the full search case but it is $C_{16}=16+16=32$ for the proposed one. Thus, it can be said that the proposed algorithm reduced the complexity significantly with a ratio of $87.5 \%$, without too much BER performance loss. In order to compare the channel estimation performance, the MSE result is also given in Fig. 6. It is seen that the EM-based schemes outperforms the initial channel estimation scheme and the frequency domain schemes achieve better MSE performance than the time domain scheme. The proposed algorithm and the full search case give almost the same results and they are both close to the Cramer-Rao Lower Bound (CRLB). Thus, it can be said that the channel estimation performance is not effected when eliminating the symbol pairs that are belong to not selected regions in the proposed algorithm. The reason is that only the symbols in the neighborhood can give valuable information for accurate estimation while the others have almost zero probability to contribute on channel estimation process. Thus, it is shown that it is not necessary to search all points in the constellation. This result is important since the complexity of the system is decreased and it is now more suitable for the practical applications.

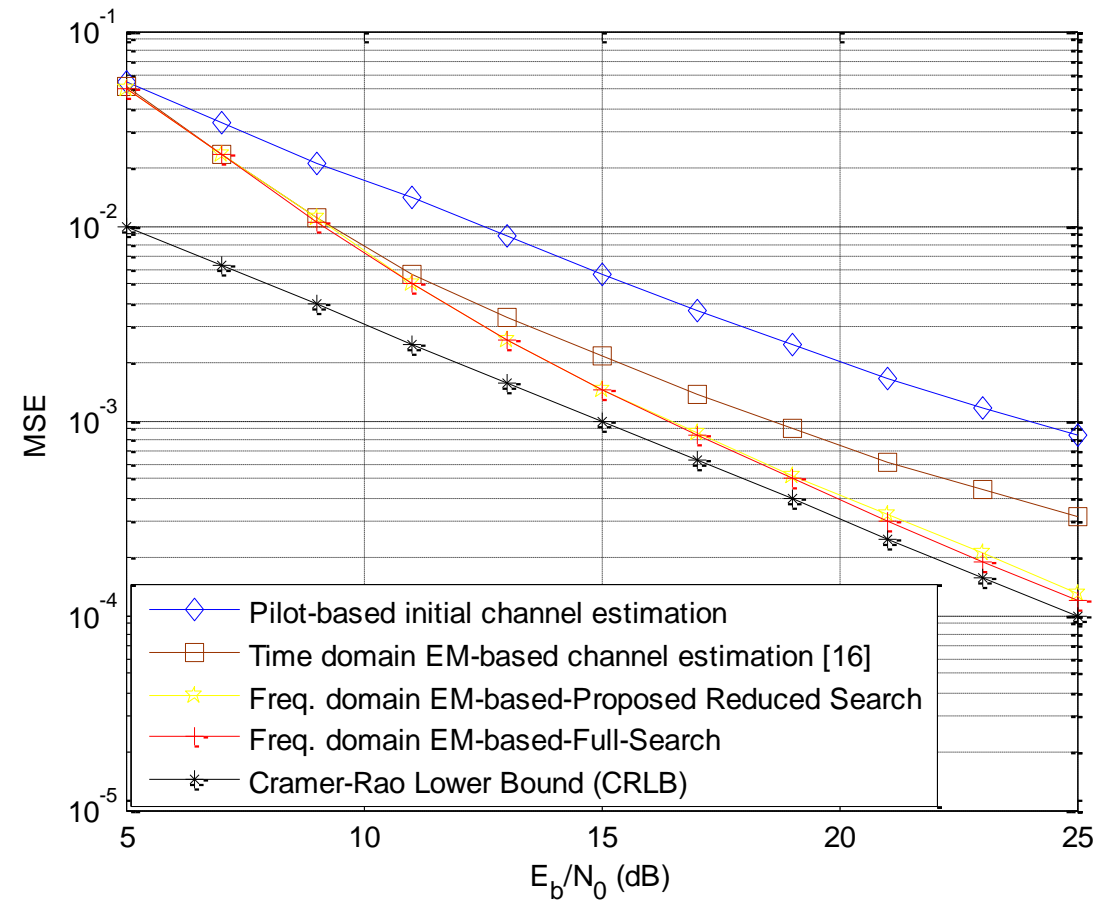

Fig. 6. The MSE performance of STBC-OFDM for 16QAM. 


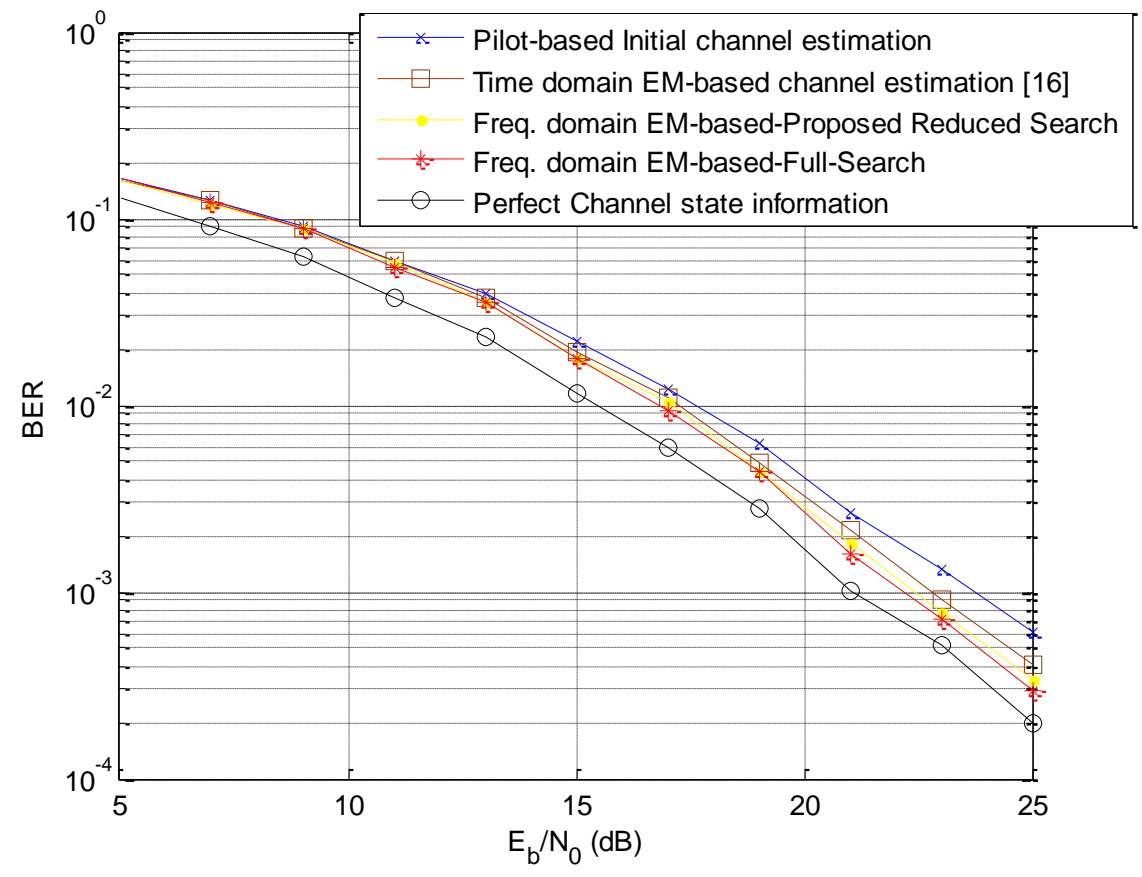

Fig. 7. The BER performance of STBC-OFDM for 64QAM.

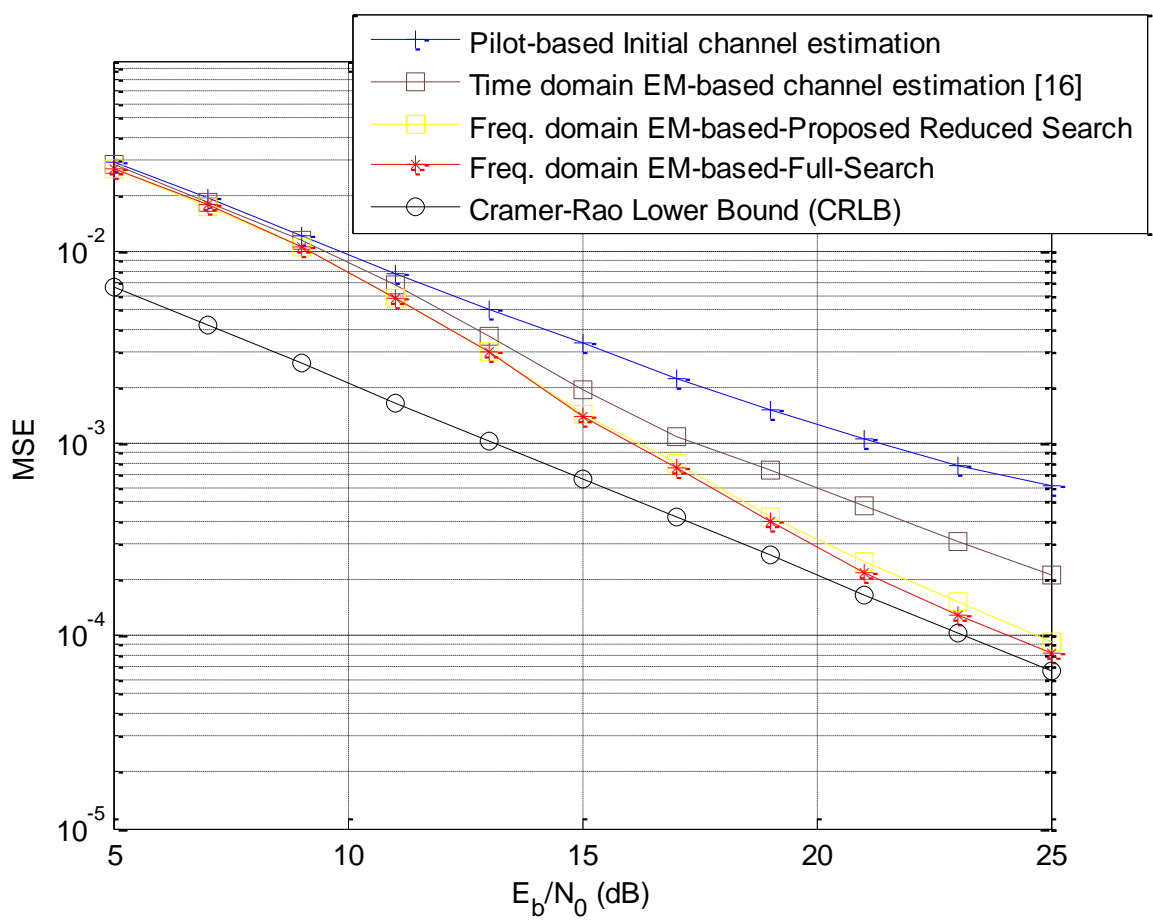

Fig. 8. The MSE performance of STBC-OFDM for 64QAM. 
Second of all, the BER and MSE performances are obtained for 64QAM in Fig. 7 and Fig. 8, respectively. It is seen that the performance of the initial channel estimation has been improved by using the EM-based channel estimation algorithms. The BER and MSE performances of the proposed reduced search case and full search case are almost the same and close to the perfect CSI and CRLB, respectively. Both of the frequency domain schemes outperform the time domain EM-based scheme for the higher order modulation schemes. For 64QAM, the total number of symbol pairs so the total calculation value is $C=4096$ for the full search case but it is $C_{64}=256+16=272$ for the proposed one. Thus, it can be seen that increasing the number of points to be searched does not effect the performance results too much. The proposed algorithm also reduces the complexity with a ratio of $93 \%$ for this case.

Table 1. Average execution time $(\mathrm{sec})$ of the algorithms $(\mathrm{Eb} / \mathrm{No}=20 \mathrm{~dB})$

\begin{tabular}{|c|c|c|}
\hline Algorithm & Full Search Case & Proposed Reduced Search Case \\
\hline Modulation & 37.8 & 3.9 \\
\hline 16QAM & 540 & 4.2 \\
\hline
\end{tabular}

Finally, the complexity of the full search case and proposed reduced search case algorithms are compared by measuring the execution time required by each of them in order to support the conclusions drawn by using BER and MSE results. The results given in Table 1 are obtained by using MATLAB R2011b on a PC with Core 2 Duo processor operating with a clock $3 \mathrm{GHz}$. These results also show that the proposed algorithm decrease the complexity of the algorithm significantly.

\section{Conclusion}

In this paper, we have proposed a low complexity frequency domain EM-based channel estimation method for STBC-OFDM with higher order constellations. The proposed scheme revealed that it is not required to search all constellation points to obtain a good performance. The complexity of the EM-based channel estimation for STBC-OFDM has been decreased significantly by reducing the number of constellation points to be searched and the process time of the channel estimation has been shortened without any performance loss. Therefore, the proposed algorithm is suitable for practical wireless communication systems with high data rate applications. Moreover, the proposed channel estimation method can be extended to multiuser MIMO-OFDM schemes with more than two transmit and receive antennas and any order of constellations.

\section{References}

[1] Cimini L., "Analysis and simulation of a digital mobile channel using orthogonal frequency multiplexing," IEEE Transactions on Communication, vol. 3, pp. 665-675,1985. Article (CrossRef Link).

[2] Alamouti S. M., "A simple transmit diversity technique for wireless communications," IEEE Journal on Select Areas in Communication, vol. 16, pp. 1451-1458, 1998.

Article (CrossRef Link). 
[3] Tarokh V, Jafarkhani H, Calderbank AR., "Space-Time Block Coding for Wireless Communications:Performance Results," IEEE Journal on Selected Areas in Communications, vol. 17, pp. 451-460,1999. Article (CrossRef Link).

[4] Tarokh V., Jafarkhani H, Calderbank AR., "Space-time block Codes from orthogonal designs," IEEE Trans. Inform. Theory, vol. 45, pp. 1456-1467, 1999. Article (CrossRef Link).

[5] Van de Beek JJ, Edfors OS, Sandell M, Wilson SK, Börjesson OP., "On channel estimation in OFDM systems," in Proc. of IEEE Vehicular Technology Conference, pp.815-819, Chicago, 1995. Article (CrossRef Link).

[6] Coleri S, Ergen M, Puri A, Bahai A., "Channel estimation techniques based on pilot arrangement in OFDM systems," IEEE Transactions on Broadcasting, Vol. 48, pp. 223-229, 2002. Article (CrossRef Link).

[7] Lee KF, Williams DB., "Pilot-Symbol Assisted Channel Estimation for Space-Time Coded OFDM Sytems," EURASIP Journal on Applied Signal Processing , vol.5, pp. 507-516, 2002. Article (CrossRef Link).

[8] Guo J, Wang D, Ran C., "Simple channel estimator for STBC-based OFDM systems," Electronics letters, vol. 39, pp.445-447, 2003. Article (CrossRef Link).

[9] Song B, Gui L, Zhang W., "Comb type pilot aided channel estimation in OFDM systems with transmit diversity," IEEE Transactions on Broadcasting ,vol. 52, pp. 50-57, 2006. Article (CrossRef Link).

[10] Dempster AP, Laird NM, Rubin DB., "Maximum likelihood from incomplete data via the EM algorithm," J. Royal Statiscal Soc.,Ser. R. , vol.39, pp. 1-38, 1977. Article (CrossRef Link).

[11] Moon TK., "The expectation-maximization algorithm," IEEE Signal Processing Magazine, vol.13, pp.47-60, 1996. Article (CrossRef Link).

[12] McLachlan G, Krishnan T., The EM Algorithm and Extensions, Second Edition. John Wiley and Sons, 2008. Article (CrossRef Link).

[13] Ma X, Kobayashi H, Schwartz SC., "EM-Based Channel Estimation Algorithms for OFDM," EURASIP Journal on Applied Signal Processing , vol. 10, pp. 1460-1477, 2004. Article (CrossRef Link).

[14] Lu B, Wang X, Li YG., "Iterative receivers for space-time block-coded OFDM systems in dispersive fading channels," IEEE Trans. Wireless Commun., vol.1, pp. 213-225, 2002. Article (CrossRef Link).

[15] Xie Y, Georghiades CN., "Two EM-Type Channel Estimation Algorithms for OFDM with Transmitter Diversity," IEEE Transactions on Communications, vol. 51, pp. 106-115, 2002. Article (CrossRef Link).

[16] Ma X, Kobayashi H, Schwartz SC., "An EM-based channel estimation algorithm for space-time and space-frequency block coded OFDM," in Proc. of IEEE ICASSP, pp. 389-392, 2003. Article (CrossRef Link).

[17] Karkhanechi HM, Levy BC., "EM-based channel estimation for space time block coded MIMO OFDM systems," in Proc. of the IEEE SIPS Conference, pp. 177-181, Canada, 2006. Article (CrossRef Link).

[18] Feder M, Weinstein E., "Parameter estimation of superimposed signals using the EM algorithm," IEEE Trans. Acoust., Speech, Signal Processing, vol. 36, pp. 477-489, 1988. Article (CrossRef Link).

[19] Basturk I, Ozbek B., "Iterative EM-Based Channel Estimation for STBC-OFDM," in Proc. of IEEE WCNC, pp. 1-6, Budapest, 2009. Article (CrossRef Link).

[20] Hassibi, B., Vikalo, H., "On Sphere Decoding algorithm. Part I, the Expected complexity," IEEE Transactions on Signal Processing, vol. 54, no.5, pp.2806-2818, 2005. Article (CrossRef Link).

[21] Studer, C., Burg, A. and Bolcskei, H., "Soft-output sphere decoding: Algorithms and VLSI implementation," IEEE Journal on Selected Areas in Communications, vol.26, no.2, pp.290-300, 2008. Article (CrossRef Link).

[22] Mohamed G. El-Mashed, Sayed El-Rabaie, "OSIC-Based SD MIMO Detection Algorithm for LTE-A Downlink Physical Layer,” Wireless Pers Commun. , vol. 80, pp. 751-768, 2015. Article (CrossRef Link). 
[23] A. Nafkha, E. Boutillon, and C. Roland, "Near maximum likelihood detection for MIMO systems using an intensification strategy over a $\mathrm{BCH}$ codes," in Proc. of International Conference on Smart Systems and Devices, Hammamet, Tunisie, 2007. Article (CrossRef Link).

[24] F. Wang, Y. Xiong, and X. Yang, "Approximate ML detection based on MMSE for MIMO systems," in Proc. of Progress in Electromagnetics Research Symposium online, pp. 475-480, 2007. Article (CrossRef Link).

[25] M. Rupp, G. Gritsch, H. Weinrichter, “Approximate ML Detection for MIMO Systems With Very Low Complexity,” in Proc. of IEEE ICASSP, pp. 809-812, May 2004. Article (CrossRef Link).

[26] N. Tax, Z. Wu, V. Dantona and B. Lankl, "Novel Two-Stage Detection for MIMO OFDM Systems with Reduced Complexity," in Proc. of International ITG Workshop on Smart Antennas, pp.1-7, Ilmenau, Germany, 2015. Article (CrossRef Link).

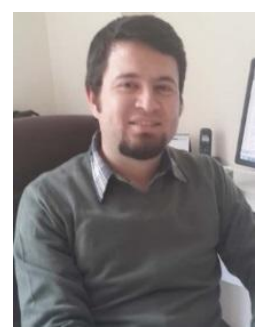

Illhan Baştürk is an Assistant Professor in Electrical and Electronics Engineering Department at Adnan Menderes University, Turkey. He graduated from Electrical and Electronics Engineering Department at Dumlupınar University, Kütahya, Turkey in 2003. He received his MSc and $\mathrm{PhD}$ degrees from Electrical and Electronics Engineering Department at Izmir Institute of Technology, İzmir, Turkey respectively on 2007 and 2014. He has been awarded with an International Doctoral Research Scholarship and he was invited to CNAM Electronics and Communication Laboratory, Paris, France in 2012. His research interests are on multiuser multiantenna OFDM systems, channel estimation techniques, radio resource management for OFDMA, relay-assisted wireless networks.

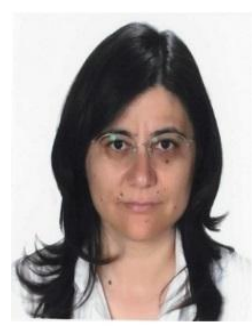

Berna Özbek is graduated from Electrical and Electronics Department of Dokuz Eylul University, Turkey and completed her MSc and $\mathrm{PhD}$ studies respectively on 1999 and 2004. Afterwards, she received a scholarship and worked as a postdoctoral researcher at CNAM-Paris on 2005. Currently, she holds an Assistant Professor position in Telecommunication field at the Electrical and Electronics Engineering Department of İzmir Institute of Technology, Turkey and is working in the field of wireless communication systems for more than fifteen years. Her research interests are on interference management strategies, resource allocation, reduced feedback strategies, multiuser multi-antenna OFDM systems and relay-based wireless systems. She has published several scientific papers at high quality journals and conferences and one book the last years. She has been awarded as a Marie-Curie Intra-European (EIF) Fellow by European Commission for two years in the project entitled Interference Management Techniques for Multicell Networks on 2010. She has completed four international projects and is currently managing two national projects as a principal coordinator. 